\title{
Souveraineté dans les colonies, souveraineté en métropole : le rôle de la Nouvelle-France dans la consolidation de l'autorité maritime en France, 1620-1628
}

\section{Helen Dewar}

Volume 64, numéro 3-4, hiver-printemps 2011

La Nouvelle-France et l'Atlantique

URI : https://id.erudit.org/iderudit/1017970ar

DOI : https://doi.org/10.7202/1017970ar

Aller au sommaire du numéro

\section{Éditeur(s)}

Institut d'histoire de l'Amérique française

ISSN

0035-2357 (imprimé)

1492-1383 (numérique)

Découvrir la revue

Citer cet article

Dewar, H. (2011). Souveraineté dans les colonies, souveraineté en métropole : le rôle de la Nouvelle-France dans la consolidation de l'autorité maritime en France, 1620-1628. Revue d'histoire de l'Amérique française, 64(3-4), 63-92. https://doi.org/10.7202/1017970ar

\section{Résumé de l'article}

Cet article traite du rôle que jouait la Nouvelle-France dans la consolidation de l'autorité maritime en France, d'abord sous l'Amirauté puis sous la Couronne, au début du XVII ${ }^{\mathrm{e}}$ siècle. L'incorporation de la colonie dans ces juridictions montre que les activités outre-mer faisaient partie des calculs dynastique et politique des titulaires de la même façon que leurs offices et commissions le faisaient en France. Ces activités en Nouvelle-France étaient susceptibles des rivalités personnelles et institutionnelles. L'extension de la juridiction amirale, dedans et hors de France, encouragée par le roi, met en relief les relations intimes entre la formation de l'État et la construction de la souveraineté coloniale dans l'Atlantique français. 


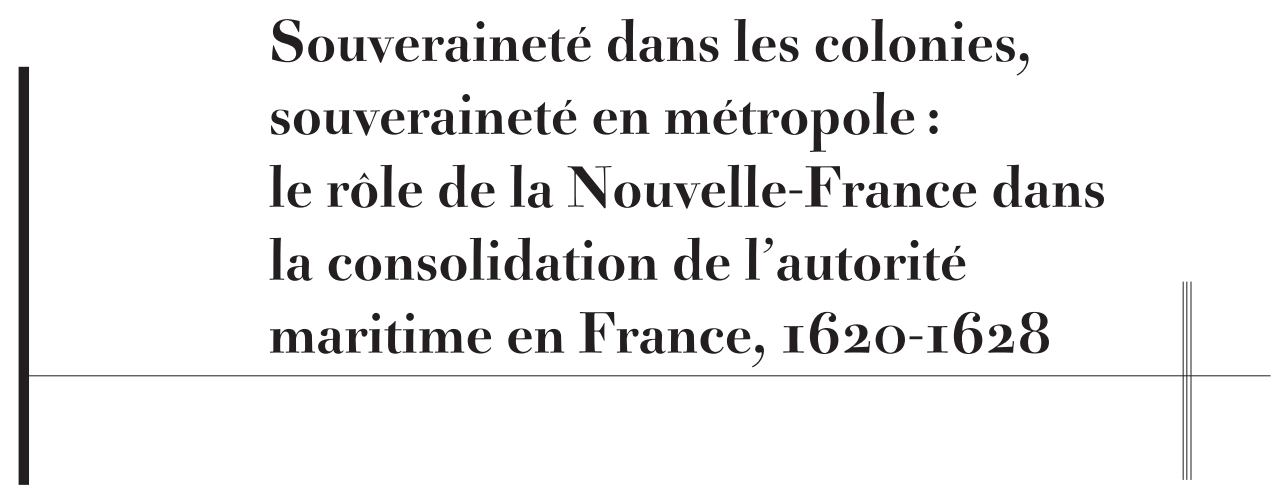

HELEN DewAR ${ }^{\mathrm{I}}$

Département d'histoire et d'études classiques

Université McGill

RÉsumÉ - Cet article traite du rôle que jouait la Nouvelle-France dans la consolidation de l'autorité maritime en France, d'abord sous l'Amirauté puis sous la Couronne, au début du $\mathrm{XVII}$ e siècle. L'incorporation de la colonie dans ces juridictions montre que les activités outre-mer faisaient partie des calculs dynastique et politique des titulaires de la même façon que leurs offices et commissions le faisaient en France. Ces activités en Nouvelle-France étaient susceptibles des rivalités personnelles et institutionnelles. L'extension de la juridiction amirale, dedans et hors de France, encouragée par le roi, met en relief les relations intimes entre la formation de l'État et la construction de la souveraineté coloniale dans l'Atlantique français.

ABSTRACT - This article examines the role played by New France in the consolidation of maritime authority in France, first under the Admiralty then under the Crown, in the early $17^{\text {th }}$ century. The colony's incorporation into these jurisdictions demonstrates that activities overseas figured into the dynastic and political calculations of titleholders in much the same way as did their positions in France and were subject to personal and institutional rivalries. Royal encouragement to Admirals to extend their jurisdictions to peripheries within and outside France highlights the intimate relations between state formation and empire building in the French Atlantic.

1. L'auteure remercie les directeurs de ce numéro, Catherine Desbarats et Thomas Wien, ainsi qu'Allan Greer, Paul Cohen et les évaluateurs anonymes de la RHAF pour leurs commentaires sur les versions antérieures de l’article. 
$\mathrm{E}^{\mathrm{n}}$ n avril 1628, une flotte de huit navires transportant 400 personnes, des vivres et les outils nécessaires pour défricher et construire une habitation quitte Dieppe et met les voiles vers la Nouvelle-France; la longue traversée de l'Atlantique se fait sous les ordres de la toute nouvelle Compagnie de la Nouvelle-France (CNF), ou Compagnie des CentAssociés. Ainsi commencent enfin, selon le récit traditionnel, la colonisation et le développement soutenus de la jeune colonie ${ }^{2}$. On peut cependant comprendre cet événement comme une sorte d'aboutissement plutôt que comme un commencement. Il constitue en effet une étape importante dans la consolidation du pouvoir maritime français qui s'amorce vers la fin du XVI ${ }^{\mathrm{e}}$ siècle. On a longtemps négligé le rôle de la NouvelleFrance dans ce processus au début du XVII ${ }^{\mathrm{e}}$ siècle. Pourtant, en l'examinant, on arrive à mieux comprendre à la fois l'histoire de l'empire français et celle de sa colonie nord-américaine durant cette période de formation.

Le «monde atlantique» est un cadre conceptuel utile pour étudier sous un nouveau jour la création de la Compagnie de la Nouvelle-France et son rôle dans la consolidation du pouvoir maritime en France. Ce cadre offre une vision plus complexe que celles proposées par les historiographies canadienne et française. D’abord, les luttes pour le pouvoir en France influencent le développement de la compagnie de colonisation. Les parties prenantes au développement de la Nouvelle-France - de l'amiral français aux vice-rois et aux lieutenants-généraux et des ministres à l'origine des compagnies aux actionnaires - regardent du même œil les affaires coloniales et les affaires métropolitaines. Dans les deux sphères, les activités portent la marque des réseaux de patronage et des calculs politiques et patrimoniaux. En outre, les nouveaux espaces, outre-mer, jouent un rôle important dans les rivalités sur le plan intérieur comme à l'échelle internationale. Les routes commerciales, la puissance navale et le contrôle des sujets au-delà des frontières traditionnelles de l'État deviennent tous des éléments clés de la réputation d'un État européen. Par extension, le contrôle de ce territoire colonial excite de plus en plus les convoitises en France, notamment au sein du gouvernement et de l'amirauté.

Cet article s'intéresse donc à la nature transatlantique de la croissance de l’autorité maritime française. J’y présente les problèmes relevés dans l’économie française et les solutions proposées, notamment la création

2. Marcel Trudel, Histoire de la Nouvelle-France (ci-après HNF) (Montréal, Fides, 1979-1983), 3 : La seigneurie des Cent-Associés, 1627-1663. 
de compagnies pour le commerce outre-mer, initiatives qui permettront aux amiraux successifs d'accroître leur pouvoir en France et dans les colonies. J'aborde également le rôle joué par la Nouvelle-France dans les rivalités personnelles et institutionnelles en France. Dans ce contexte, l'histoire des origines de la CNF devient une lentille à travers laquelle on peut voir les liens étroits entre la formation de l'État dans la métropole et la construction d'un empire à l'étranger. Loin d'être séparés, ces deux processus s'entrecroisent, ce qui n'est nulle part aussi évident que dans les domaines du commerce maritime et de la puissance navale durant les années 1620 .

\section{LE CONTEXTE HISTORIOGRAPHIQUE}

Afin de définir «la formation de l’État», il faut d’abord définir «l’État», entreprise difficile en elle-même. Les spécialistes définissent l’État selon ses institutions, militaires, financières et administratives, ou selon ses fonctions, le plus souvent celles d'un organisme de contrôle social ou moral $^{3}$. Mais ces deux méthodes situent le pouvoir d'initiative au centre. Je me range avec ceux qui vont au-delà d'un cadre institutionnel restreint pour intégrer à l'analyse «l'ensemble des acteurs exerçant le pouvoir politique», depuis le fonctionnaire le plus modeste jusqu'au roi en personne ${ }^{4}$. Cette approche, qui englobe à la fois les contextes locaux et le centre, évite la tendance de réifier l'État et de le voir comme une entité autonome qui exerce son pouvoir sur une société plutôt passive ${ }^{5}$.

Parallèlement aux visions institutionnelle et fonctionnelle de l'État, il existe deux perspectives traditionnelles sur la croissance de l'appareil étatique à l'époque moderne. La première souligne l'importance du dispositif fiscal et militaire : l’État, assumant un fardeau de plus en plus lourd à cause de la multiplication des conflits, développe une administration

3. Allan Greer et Ian Radforth, "Introduction», dans Allan Greer et Ian Radforth, dir., Colonial Leviathan: State Formation in Mid-Nineteenth-Century Canada (Toronto, University of Toronto Press, 1992), 10; Michael J. Braddick, State Formation in Early Modern England: c.1550-1700 (Cambridge, Cambridge University Press, 2000), 14-15; Timothy Mitchell, «Limits of the State: Beyond the Statist Approaches and Their Critics", American Political Science Review, 85,1 (1991): 76-78.

4. M. J. Braddick, State Formation..., op. cit., 6; John Brewer et Eckhart Hellmuth, «Introduction: Rethinking Leviathan", dans John Brewer et Eckhart Hellmuth, dir., Rethinking Leviathan : the EighteenthCentury State in Britain and Germany (Oxford, Oxford University Press, 1999), 20.

5. Michael J. Braddick, «State Formation and Social Change in Early Modern England: A Problem Stated and Approaches Suggested ", Social History, 16,1 (janvier 1991): 6. Sur l'autonomie de l'État comme réalité et comme illusion, voir T. Mitchell, «Limits of the state... », loc. cit., 81-89, 91-95. 
fiscale plus élaborée afin de mobiliser les ressources nécessaires ${ }^{6}$. La deuxième évoque un "phénomène culturel» qui correspond à l'infiltration de l'État dans diverses sphères de la vie ${ }^{7}$. Il existe une forte tendance à assimiler la formation de l'État à la création de formes bureaucratiques de gouvernement. À l'époque moderne, cependant, la «cooptation des élites ", grâce aux réseaux de patronage et de clientélisme, aux privilèges, aux monopoles commerciaux, à l'affermage des impôts et à l'attribution des fonctions officielles, fut un élément crucial dans l'accroissement de la portée de l'État ${ }^{8}$. Cette manière de comprendre la formation de l'État met au premier plan sa caractéristique principale: elle résulte des actions d'une multitude d'acteurs plutôt que d'une "volonté centrale ${ }^{9}$ ». Ainsi, son évolution n'est pas linéaire, mais sujette à des contestations, des reculs, des imprévus et des accommodements.

Plusieurs spécialistes du monde britannique et anglo-américain ont commencé à étudier le lien entre la formation de l'État et le prolongement de la souveraineté sur des terres coloniales et ils ont soutenu qu'il s'agissait de processus interdépendants et non parallèles ${ }^{10}$. À ce jour, les historiens de la présence française dans le monde atlantique se sont montrés moins enclins à traiter du sujet ${ }^{11}$. Dans le cas britannique, ce lien est peut-être plus visible du fait que les querelles internationales résultant d'activités coloniales demandent l'intervention de la Couronne, ce qui place les colonies sous la rubrique de la politique étrangère et donc sous la prérogative de la Couronne. Dans le cas français, la pratique royale traditionnelle d'accorder une vaste gamme de privilèges à des individus et à des

6. John Brewer, The Sinews of Power: War, Money, and the English State, 1688-1783 (Boston, Unwin Hyman, 1989), xiii-xvii; Michael J. Braddick, «The English Government, War, Trade, and Settlement, 16251688 ", dans William Roger Louis et Nicholas Canny, dir., The Oxford History of the British Empire (Oxford, Oxford University Press, 1998), 1: 287-292; Thomas Ertman, Birth of the Leviathan: Building States and Regimes in Medieval and Early Modern Europe (Cambridge, Cambridge University Press, 1997), 4, 6-16.

7. A. Greer et I. Radforth, «Introduction», op. cit., 10; M. J. Braddick, State Formation..., op. cit., 13-16.

8. Elizabeth Mancke et John G. Reid, «Elites, States, and the Imperial Contest for Acadia», dans John G. Reid, dir., The "Conquest» of Acadia, 1710 : Imperial, Colonial, and Aboriginal Constructions (Toronto, University of Toronto Press, 2004), 29; M. J. Braddick, State Formation..., op. cit., 24-39; T. Ertman, Birth of the Leviathan..., op. cit., 28; J. Brewer, The Sinews of Oower..., op. cit., 12-17.

9. M. J. Braddick, State formation..., op. cit., 47.

10. M. J. Braddick, State formation..., op. cit., chap. 9; Elizabeth Mancke, "Empire and State», dans David Armitage et Michael J. Braddick, dir., The British Atlantic World, 1500-1800 (New York, Palgrave Macmillan, 2009), 193-213, deuxième édition; E. Mancke et J. G. Reid, «Elites, States, and the Imperial Contest for Acadia... », loc. cit., 25-47; James Muldoon, Empire and Order: the Concept of Empire, 800-1800 (New York, St. Martin's Press, 1999), en particulier 8, 145-146.

11. Catherine Desbarats, "La question de l'État en Nouvelle-France», dans Philippe Joutard et Thomas Wien, dir., Mémoires de la Nouvelle-France. De France en Nouvelle-France (Rennes, Presses universitaires de Rennes, 2005), 187-198. 
sociétés pour assurer la loyauté dans tout le royaume fait en sorte que les rapports entre détenteurs de titres dans les colonies sont très souvent liés à l'octroi et à l'exercice de pouvoirs et de privilèges dans la métropole ${ }^{12}$. L'édification de la souveraineté en terre coloniale, tout comme le processus de formation de l'État, ne se limite pas à l'implantation d'une autorité absolue. Au contraire, les innombrables prétentions, revendications et contestations de la part de sujets, de concurrents étrangers et de peuples autochtones maintiennent l'empire dans un perpétuel «état de devenir ${ }^{13}$ ». Autant les activités des sujets sur le continent européen favorisent la formation de l'État, autant celles des sujets dans les colonies incitent l'État à s'impliquer de plus en plus dans l'expansion coloniale ${ }^{14}$.

C'est peut-être dans la lutte de la France pour le contrôle de la mer, au début du XVII ${ }^{\text {e }}$ siècle, que les deux processus - formation de l'État et construction de l'empire - convergent de la façon la plus évidente. Cette lutte se déroule à plusieurs niveaux: en France, contre les huguenots, établis notamment à La Rochelle, qui contrôlent la côte atlantique - avec l'aide de leurs coreligionnaires d'Angleterre; sur le plan international, contre les Néerlandais et les Anglais qui tirent profit de l'expansion de leurs routes commerciales et de leur pouvoir maritime; et enfin outremer, contre les sujets qui essaient de voyager librement entre la France et des colonies comme la Nouvelle-France à l'insu des autorités maritimes de la métropole ${ }^{15}$. Importante d'un point de vue stratégique, la mer est donc aussi un espace que l'on se dispute et qui alimente les rivalités en France, entre la couronne et l'amirauté, entre l'amiral et les ministres et parmi les sujets ayant des intérêts dans les colonies, qui tentent tous de s'accaparer les richesses et le prestige de ce domaine immense.

12. M. J. Mancke et J. G. Reid, «Elites, the State, and the Imperial Contest for Acadia... », loc. cit., 29; E. Mancke, «Empire and State...», loc. cit., 207.

13. Ann Laura Stoler, "On Degrees of Imperial Sovereignty», Public Culture, 18,1 (2006): 135-136; C. Desbarats, "La question de l'État», loc. cit., 189; Frederick Cooper et Ann Laura Stoler, "Between Metropole and Colony", dans Frederick Cooper et Ann Laura Stoler, dir., Tensions of Empire: Colonial Cultures in a Bourgeois World (Berkeley, University of California Press, 1997), 3-4. Sur le rôle des prétentions et revendications contradictoires dans l'instabilité des régimes politiques, voir Lauren Benton, A Search for Sovereignty: Law and Geography in European Empires, 1400-1900 (Cambridge, Cambridge University Press, 2010), en particulier chap. 1; James J. Sheehan, "The Problem of Sovereignty in European History", American Historical Review, 111,1 (février 2006): 1-15.

14. Elizabeth Mancke, "Chartered Enterprise and the Evolution of the British Atlantic World", dans Elizabeth Mancke et Carole Shammas, dir., The Creation of the British Atlantic World (Baltimore, Johns Hopkins University Press, 2005), 239, 262.

15. Sur l'argument selon lequel la «double mission» de La Rochelle et de la Nouvelle-France convergent dans les domaines «du commerce et du transport », voir J. F. Bosher, "The Political and Religious Origins of La Rochelle’s Primacy in Trade with New France, 1627-1685 », French History, 7,3 (1993): 286312, citation 295. 
Dans ce contexte, la Nouvelle-France - telle qu'on se l'imagine, plus que pour ce qu'elle est, une petite colonie en difficulté - revêt une importance particulière, autant sur le plan stratégique que sur celui de l'ambition personnelle ${ }^{16}$. L'histoire de la création de la Compagnie de la Nouvelle-France fait partie d'un récit plus vaste exposant l'importance grandissante de la puissance navale de la France et de son commerce maritime. L'historiographie de la Nouvelle-France au début du XVII siècle, qui s'est traditionnellement concentrée sur le développement de l'administration coloniale et de la colonisation, s'est fort peu intéressée à ces questions. Les historiens Marcel Trudel et H. P. Biggar, par exemple, ont examiné les activités des détenteurs de titres et de droits divers en Nouvelle-France, qu'il s'agisse d'individus ou de compagnies, à la lumière des obligations et privilèges stipulés dans les commissions royales ${ }^{17}$. Tous deux ont estimé que des échecs successifs avaient émaillé les décennies antérieures à 1627, pour diverses raisons: Couronne inconstante, marchands avares, conflits entre catholiques et protestants et obligations trop lourdes. Leurs ouvrages renferment l'affirmation implicite selon laquelle la colonisation aurait dû se poursuivre d'une certaine façon. D'autres historiens, dont Gustave Lanctot et Lucien Campeau, décrivent la mise en place d'une infrastructure administrative et financière durant le mandat de la CNF et au-delà ${ }^{18}$. Toutes ces études font pratiquement abstraction du contexte français qui entoure les événements coloniaux qu'elles décrivent.

Témoins de l'intérêt croissant pour le cadre atlantique manifesté par les historiens de l'empire français, plusieurs études récentes abordent le contexte politique métropolitain entourant la colonisation ${ }^{19}$. David Hackett Fischer décrit les initiatives de Samuel de Champlain pour bâtir des réseaux d'appui à l'effort de colonisation auprès de la cour, au sein du gouvernement et parmi les communautés religieuses. Gervais Carpin affirme que la Compagnie de la Nouvelle-France est liée de façon inextricable à la "politique maritime» du cardinal de Richelieu ${ }^{20}$. En se servant

16. Sur l'idée que les colonies sont à la fois des "espaces imaginaires et physiques", voir Cooper et Stoler, «Between Metropole and Colony... », op. cit., 3.

17. Marcel Trudel, HNF (Montréal, Fides, 1963-1983), v. 1-3; H. P. Biggar, The Early Trading Companies of New France, 1534-1632 (Michigan, Scholarly Press, [1901] 1972).

18. Gustave Lanctot, L'administration de la Nouvelle-France (Montréal, Éditions du jour, 1971); Lucien Campeau, Les finances publiques de la Nouvelle-France sous les Cent-Associés, 1632-1665 (Montréal, Bellarmin, 1975).

19. Sur l'Atlantique français comme cadre conceptuel, voir Silvia Marzagalli, "The French Atlantic», Itinerario, 23,2 (1999): 70-83.

20. David Hackett Fischer, Le rêve de Champlain (Montréal, Boréal, 2011); Gervais Carpin, Le Réseau du Canada: Étude du mode migratoire de la France vers la Nouvelle-France (1628-1662) (Paris, Presses de l'Université de Paris-Sorbonne, 2001). 
de ses réseaux de clients, Richelieu a créé et gardé sous son emprise la $\mathrm{CNF}$ et d'autres entreprises de commerce et de colonisation. Si Carpin apporte des précisions très utiles sur le contexte métropolitain derrière la $\mathrm{CNF}$, il accorde presque tout le mérite à un individu, Richelieu lui-même. La forte tendance historiographique à voir les entreprises de la NouvelleFrance des décennies antérieures comme des échecs renforce l'image du cardinal en tant que pionnier, voire de sauveur. Cependant, l'approche de Richelieu ne faisait que reconduire, de bien des façons, les mesures centralisatrices adoptées sous les amiraux de France.

Le débat récurrent au sein de l'historiographie française sur l'approche de Richelieu au gouvernement est pertinent ici. Richelieu y est traditionnellement dépeint comme un réformateur avec un programme cohérent et de longue durée visant à rendre l'administration gouvernementale plus bureaucratique $^{21}$. Des études récentes avancent plutôt qu'il réagit selon les circonstances et qu'il travaille de façon pragmatique au sein des institutions existantes ${ }^{22}$. Dans cet ordre d'idées, je soutiens que les initiatives maritimes du cardinal font partie d'une tendance plus longue vers une autorité personnelle amplifiée chez ceux qui sont chargés de surveiller le commerce maritime et la navigation, ce qui a pour effet secondaire de prolonger l'autorité de la Couronne vers la périphérie du royaume. Si l'emprise de cette dernière s'accroît ainsi, elle le fait par l'entremise de réseaux de patronage autant que par de nouvelles fonctions administratives. Ces pratiques correspondaient à la fois aux intérêts du roi et aux intérêts patrimoniaux des détenteurs de titres et de droits divers ${ }^{23}$. Le prolongement de l'autorité maritime vers la Nouvelle-France sous Charles

21. Voir, par exemple, Victor-L. Tapié, France in the Age of Louis XIII and Richelieu (Cambridge, Cambridge University Press, 1984) traduction D. McN. Lockie, deuxième édition; J. H. Elliott, Richelieu et Olivares (Cambridge, Cambridge University Press, 1984); Pierre Castagnos, Richelieu face à la mer (Éditions Ouest-France, 1989).

22. Voir, entre autres, Robin Briggs, "Richelieu and Reform: Rhetoric and Political Reality", dans Joseph Bergin et Laurence Brockliss, dir., Richelieu and His Age (Oxford, Clarendon Press, 1992), 71-97; David Parrott, Richelieu's Army: War, Government and Society in France, 1624-1642 (Cambridge, Cambridge University Press, 2001); Alan James, The Navy and Government in Early Modern France, 1572-1661 (Rochester, NY, Royal Historical Society and Boydell Press, 2004); L.-A. Boiteux, Richelieu, grand maître de la navigation et du commerce de France (Paris, Ozanne, 1955).

23. J’emploie l'expression "autorité personnelle» pour décrire l'exercice du pouvoir au moyen de liens personnels et de réseaux de patronage et de clientélisme, par opposition à un système bureaucratique de gouvernement dans lequel les postes sont attribués selon le mérite et définis selon des fonctions spécifiques. Voir Alan James, The Origins of French Absolutism, 1598-1661 (Harlow, Pearson Longman, 2006), 61-63; Sharon Kettering, Patrons, Brokers, and Clients in Seventeenth Century France (Oxford University Press, 1986); William Beik, Absolutism and Society in Seventeenth-Century France: State Power and Provincial Aristocracy in Languedoc (Cambridge, Cambridge University Press, 1989); David Parker, "Class, Clientage, and Personal Rule in Absolutist France», Seventeenth-Century French Studies, 9 (1987): 192-213. 
et Henri de Montmorency, amiraux de France, puis sous Richelieu, grand maître, chef et surintendant de la navigation et du commerce de France, démontre l'importance relative de la colonie et sa valeur à la fois stratégique et dynastique.

\section{LE CONTEXTE HISTORIQUE: LES AFFIRMATIONS DE LA SOUVERAINETÉ FRANÇAISE EN AMÉRIQUE DU NORD JUSQU'EN 1627}

Même si la France lance des tentatives de colonisation en Amérique du Nord au cours du $\mathrm{XVI}^{\mathrm{e}}$ siècle, c'est seulement à la fin des guerres de religion, en 1598, que se fait un travail concerté pour consolider par ce moyen les prétentions françaises à une grande partie du continent. Incapable d'absorber les coûts et les risques considérables associés à de telles aventures, ou non disposée à le faire, la Couronne française, à l'instar de ses rivales, engage des entreprises privées à cette fin. Alors que des compagnies par actions établissent plusieurs des premières colonies anglaises en Amérique, des propriétaires entreprennent les premières tentatives françaises. Un propriétaire est un individu, souvent de la noblesse, qui obtient une commission du roi pour exercer des pouvoirs gouvernementaux sur un territoire. Ces pouvoirs comprennent habituellement le droit de nommer des capitaines et des officiers de justice, de distribuer des terres et de maintenir une armée ${ }^{24}$. En accordant des pouvoirs royaux aussi vastes, le roi veut que le propriétaire et ses hommes, par leurs activités d'exploration, de commerce et de colonisation, prolongent son autorité souveraine sur le plus grand territoire possible. Les aspirations du roi expliquent en partie les dimensions, souvent très vastes, des territoires accordés à un propriétaire; en pratique, le contrôle réel de la France se limite à un territoire très restreint.

Après plusieurs tentatives de colonisation infructueuses, Pierre du Gua, sieur de Monts, reçoit deux commissions en 1603 : la première, de l'amiral de France, Charles de Montmorency, le nomme vice-amiral de NouvelleFrance et la deuxième, d'Henri IV, le nomme lieutenant-général de la colonie. Ces pouvoirs s'accompagnent de la responsabilité de peupler le territoire; enfin, pour compenser les coûts associés à la colonisation, de Monts reçoit le droit exclusif de la traite pendant dix ans. Il établit une association commerciale avec des marchands de Rouen, de La Rochelle

24. Même si on peut voir la concession de territoires outre-mer à des compagnies ou à des individus comme des formes de propriété, le terme "propriétaire» ne renvoie habituellement qu'à un individu. Sur la propriété définie de façon large, voir L. H. Roper et Bertrand van Ruymbeke, «Introduction", dans L. H. Roper et B. van Ruymbeke, dir., Constructing Early Modern Empires: Proprietary Ventures in the Atlantic World, 1500-1750 (Leiden, Brill, 2007), 2-3, 7-9. 
et de Saint-Malo, ayant pour but d'équiper des navires sur une base annuelle, avec de la marchandise et des provisions pour la colonisation, afin de faire la traite avec les groupes autochtones et de recruter de nouveaux colons. La compagnie établit deux colonies en Acadie, avant de fonder Québec en 1608, sous les ordres du lieutenant de De Monts, Samuel de Champlain. Pour sa part, de Monts passe le plus clair de son temps à faire pression à la cour. Il perd ses privilèges plus tôt que prévu en raison d'une opposition féroce en France, ce qui ouvre une période de libre-échange entre 1609 et 1612 . Sa situation se détériore davantage avec la mort, en 1610, de l'un de ses deux grands protecteurs, Henri IV, et la démission de l'autre, Charles de Montmorency, en 1612. Peu après, il perd ses titres de vice-amiral et de lieutenant-général de la Nouvelle-France ${ }^{25}$.

Afin de poursuivre le développement de l'habitation de Québec, de Monts et Champlain estiment qu'il leur faut un défenseur bien placé et capable d'influencer la cour et le jeune Louis XIII. Après un faux départ, le roi nomme Henri de Bourbon, prince de Condé, lieutenant-général de la Nouvelle-France en novembre 1612. Bien qu'il possède les mêmes pouvoirs et privilèges que son prédécesseur, Condé exerce ses fonctions de lieutenant-général de façon radicalement différente. Prince héréditaire, gouverneur et lieutenant-général de Guyenne, Condé n’a aucun intérêt particulier pour la Nouvelle-France : c'est plutôt sa relation avec le roi qui explique sa nomination. Son rôle est donc surtout symbolique, qui témoigne de l'engagement de la Couronne envers l'occupation de la Nouvelle-France. Condé délègue son autorité administrative à son lieutenant, Champlain, et ses privilèges commerciaux à la Compagnie de Rouen et Saint-Malo (CRSM), une association de marchands des deux ports.

Durant le mandat de Condé se met en place le mode général de gouvernance qui prévaudra pendant quinze ans: deux autorités déléguées dotées de compétences distinctes, mais se recouvrant partiellement. C'est également sous son mandat que Québec et l'Acadie sont séparés pour relever de deux entreprises distinctes: dorénavant, les représentants du vice-roi concentreront leurs efforts sur le développement de Québec et de la vallée du Saint-Laurent. À chaque changement de lieutenant-général - ou de vice-roi, comme on l'appellera par la suite -, Champlain reçoit une nouvelle commission. En 1620, sous le duc de Montmorency, la

25. Il semble y avoir un chevauchement entre la commission reçue par de Monts à titre de vice-amiral et celle de Charles de Biencourt, sieur de Saint-Just, qui devient vice-amiral vers 1611. Voir Gabriel Marcel, dir., Factum du procès entre Jean de Biencourt, Sieur de Poutrincourt et les pères Biard et Massé, Jésuites (Paris, Maisonneuve et Charles Leclerc, 1887). 
CRSM perd ses privilèges au profit d'une nouvelle compagnie dirigée par Guillaume de Caen, capitaine de la marine royale. La nouvelle Compagnie de Montmorency continue de fonctionner malgré la nomination d'un nouveau vice-roi, en 1625. En 1627, le cardinal de Richelieu, principal ministre de Louis XIII, s'implique personnellement dans la création de la Compagnie de la Nouvelle-France, sur le modèle des compagnies néerlandaise et anglaise des Indes orientales. On accorde à la nouvelle compagnie l'autorité administrative, judiciaire, commerciale et seigneuriale partout dans la vallée du Saint-Laurent et en Acadie ${ }^{26}$. La colonie se trouve alors pour la première fois sous la gouverne directe d'une compagnie. L'évolution de la constitution et de la distribution de l'autorité tout au long de la période témoigne à la fois des expériences antérieures en Nouvelle-France et du climat politique et économique particulier en France.

\section{LE MALAISE ÉCONOMIQUE FRANÇAIS ET LES RÉFORMES PROPOSÉES POUR LE COMMERCE MARITIME}

Durant le premier tiers du XVII ${ }^{\mathrm{e}}$ siècle, un grand intérêt se manifeste, au sein du gouvernement et à l'extérieur de celui-ci, pour la recherche de solutions aux problèmes économiques qui affligent alors la France. La Couronne reçoit de nombreux mémoires sur le sujet, dont plusieurs sont anonymes et certains ont été sollicités par Richelieu ${ }^{27}$. Ces mémoires partent tous du principe que l'approche française en matière de commerce, en particulier le commerce maritime et extérieur, est totalement inadéquate, de sorte que la France traîne loin derrière ses rivaux. Pour de nombreux observateurs, le plus grand obstacle à surmonter est le mépris et la méfiance des Français à l'égard du commerce même. Un auteur signale la différence entre les attitudes en France et dans les autres pays: en Angleterre, en Espagne et ailleurs, «le trafficq et ceux qui l'exercent, n'y sont ilz pas en honneur et en estime, et ny jouissent ilz pas d'une infinité d'exemptions, et previleges? Asseurement ${ }^{28} »$. En France, par contre, affirment les observateurs, on a laissé le commerce tomber en ruine.

26. «Acte pour l'établissement de la Compagnie des Cents-Associés pour le commerce du Canada... le 29 Avril, 1627 ", dans Édits, ordonnances royales, déclarations et arrêts du Conseil d'État du Roi, concernant le Canada (Québec, P. E. Desbarats, 1803-1806), 1-17.

27. La difficulté de mettre en œuvre des solutions et d'atteindre les résultats voulus est démontrée par la longue période durant laquelle cette constellation d'idées persiste. Les mêmes problèmes et les mêmes solutions sont décrits dans des mémoires de l'autre grande période de débats sur le commerce d'outre-mer au XVII ${ }^{\mathrm{e}}$ siècle, le mandat de Jean-Baptiste Colbert.

28. Bibliothèque nationale de France (ci-après $\mathrm{BnF}$ ), département de manuscrits (ci-après dépt. de ms.), fonds français, v. 18781, f. 228, Jean Le Cler, "Advis et tres humbles remonstrances au roy sur l'establissement du commerce en France», 30 décembre 1620. 
Les Français, notamment les nobles, refusent de toucher à ce domaine. Contrairement à l'Angleterre, elle ne possède aucune tradition voulant qu'un membre de chaque famille noble choisisse une carrière maritime ${ }^{29}$. Les conséquences pratiques de ce manque d'intérêt pour les activités commerciales, notent les auteurs des mémoires, sont que la majeure partie du commerce international français est aux mains d'étrangers, en particulier des Néerlandais. Les quelques navires français qui prennent la mer sont vulnérables aux pirates et aux rivaux, étant donné l'absence d'une marine royale française. Et tandis que la France accueille toutes sortes de commerçants étrangers, les marins français sont maltraités dans les ports étrangers, particulièrement en Espagne ${ }^{30}$.

Les interactions entre la puissance maritime, les colonies et le commerce sont à la base des solutions proposées à la stagnation maritime et commerciale de la France. Différents auteurs de mémoires proposent la formation d'une marine royale et d'une marine marchande grâce aux contributions des villes, l'augmentation des droits de douane sur le commerce étranger, l'anoblissement de marchands et l'exemption à la dérogeance pour les membres de la noblesse qui s'adonnent au grand commerce maritime ${ }^{31}$. Plusieurs des mesures proposées s'inspirent de modèles étrangers. Le poète et dramaturge Antoine de Montchrestien recommande d'accorder aux constructeurs navals une prime par tonne de capacité, comme en Angleterre. Jean Eon, carme de Nantes et auteur du célèbre traité Le Commerce honorable, évoque les colonies établies par les Espagnols, les Anglais et les Néerlandais qui ont grandement augmenté la richesse de ces pays et l'étendue des territoires sous leur domination ${ }^{32}$.

29. P. Castagnos, Richelieu face à la mer..., op. cit., 119. Les contemporains n'attribuent pas le manque d'intérêt au sein de la noblesse à la France au complet, mais aux régions occidentales et septentrionales où le commerce transatlantique et international est en croissance. En France méridionale, toutes les couches de la société participaient au commerce maritime et au long cours. Voir L.-A. Boiteux, Richelieu, grand maître..., op. cit., 200.

30. BnF, dépt. de ms., fonds nouvelles acquisitions françaises, v. 7287, «Mémoire pour l'establissement du commerce en France», n.d., fo. 24v-25; Antoine de Montchrestien, Traité de l'économie politique (Paris, 1615), 249-251.

31. BnF, dépt. de ms., fonds français, v. 18781, fo. 234; BnF, dépt. de ms., fonds français, v. 17329, "Mémoire pour les conjonctions des mers», fo. 514; "Mémoire de Chevalier de Razilly», 27 novembre 1626, dans Léon Deschamps, Un colonisateur du temps de Richelieu (Paris, Institut Géographique de Paris and Charles Delagrave, 1887), 24-32; Jean Eon, Commerce honorable (Paris, 1646), 170-175, 294-295; Pierre Grillon, dir., Les Papiers de Richelieu: Section politique intérieure correspondance et papiers d'état (Paris, Éditions A. Pedone, 1975), v. 1, no. 333, 340.

32. A. de Montchrestien, Traité de l'économie politique..., op. cit., 311-312. Sur les origines des subventions pour la construction navale en Angleterre, voir David Loades, England's Maritime Empire: Seapower, Commerce and Policy, 1490-1690 (Londres, Longman, 2000), 30. J. Eon, Commerce honorable..., op. cit., 294295. L'intérêt d'un écrivain et d'un carme pour le sujet du commerce français demande une certaine 
Cependant, le principal outil emprunté aux pays avoisinants est la compagnie commerciale. Pour plusieurs auteurs de l'époque, la Compagnie néerlandaise des Indes orientales est un exemple des richesses dont la France pourrait jouir si les marchands français mettaient en commun leurs capitaux et se partageaient les risques. Non seulement les Néerlandais contrôlent le commerce des Indes et ainsi le marché des épices en Europe, mais ils attirent également des investissements étrangers dans l'entreprise, ce qui réduit les ressources disponibles pour d'éventuels concurrents ${ }^{33}$. Eon fait l'esquisse des approches espagnole, portugaise, anglaise et néerlandaise au commerce, tant en Europe qu'à l'extérieur: «il importe de bien remarquer que tout leur Négoce se fait en Compagnie, et que si tost qu'ils découvrent quelque moyen de profiter au Négoce, ils visent incontinant à former une Compagnie, faire fond, et établir l'ordre et œeconomie qu'ils veulent observer: et par ce moyen aussi ils réussissent en tout ce qu'ils entreprennent ${ }^{34} »$. Si la France adoptait une méthode progressive semblable, si les compagnies devenaient chose courante, la France connaîtrait un succès comparable. Le défi est de surmonter la propension des marchands français à faire du commerce individuellement ou dans le cadre d'associations de courte durée avec des parents et des amis ${ }^{35}$. Il faut, dit-

explication. Montchrestien a vécu en Angleterre, où il a observé le travail de Français comme lui et s'est familiarisé avec le fonctionnement de diverses industries. Il s'est aussi rendu aux Pays-Bas, où il s'est renseigné au sujet des grandes compagnies commerciales. De retour en France, il établit son propre atelier sidérurgique et une manufacture d'instruments en acier. En 1615, il rédige son traité sur l'économie politique, qu'il dédie à Louis XIII et la reine régente. Même s'il obtient une audience devant le roi et la régente, il ne réussit pas à les convaincre de suivre son programme. Désabusé, il s'allie aux huguenots (bien qu'il soit catholique) dans leur révolte. Voir Théophile Funck-Brentano "Introduction", Traicté de l'œeconomie politique (Paris, E. Plon, Nourrit, 1889), x-xxi. Jean Eon publie son traité sous couvert de l'anonymat en 1646. Contrairement à Montchrestien, qui n'avait pas d'alliés politiques forts, Eon avait plusieurs protecteurs puissants. Prieur d'une maison de carmes à Nantes, il était sous la protection du commissaire général de l'ordre du Mont-Carmel, Antoine de la Porte, lui-même parent de Meilleraye, gouverneur et lieutenant-général de la Bretagne et apparenté à Richelieu. Eon devient conseiller de Meilleraye. Une tentative des marchands de Nantes pour établir une bourse commune dans les années 1640, semblable aux premières tentatives d'établir des compagnies sous Richelieu, se heurte à l'opposition du Parlement de Rennes ainsi que d'une publication anonyme. Meilleraye, partisan du projet en question, voit la nécessité de répondre à cette opposition et a probablement proposé le nom d'Eon, écrivain qu'il sait bien placé pour formuler une riposte. Voir Charles Dugast-Matifeux, Le "Commerce honorable» et son auteur (Nantes, A. Guéraud, 1857), 6-27.

33. Évidemment, les Français ne sont pas les seuls à s'inspirer du modèle néerlandais à l'époque. Des intellectuels anglais étudient de près l'économie néerlandaise afin de repérer, pour ensuite les copier, les pratiques qui expliquent cette réussite. Plusieurs magnats londoniens tentent également d'établir une compagnie des Indes occidentales sur le modèle néerlandais. Voir Joyce Oldham Appleby, Economic Thought and Ideology in Seventeenth-Century England (Princeton, Princeton University Press, 1978), chap. 4; John C. Appleby, " An Association for the West Indies? English Plans for a West India Company 1621-29", The Journal of Imperial and Commonwealth History, 15 (1987): 213-241.

34. J. Eon, Commerce honorable..., op. cit., 295.

35. L.-A. Boiteux, Richelieu, grand maître..., op. cit., 204. 
on, des mesures incitatives, allant des exemptions sur les droits de douane à l'anoblissement des marchands, pour changer façons de faire traditionnelles du commerce français.

À quoi ressemble une compagnie établie suivant les modèles néerlandais et anglais? Les caractéristiques essentielles des sociétés par actions émergentes sont la mise en commun de capitaux et la constitution en personne morale, de sorte que la compagnie peut acquérir des biens meubles ou immeubles et agir en justice ${ }^{36}$. Le capital est au centre de l'entreprise et il est divisé en actions ("parts et portions») négociables. À l'opposé, le type d'association prédominant en France à l'époque est organisé autour des personnes qui en font partie: si un partenaire se retire, l'association se dissout ${ }^{37}$. En théorie, selon le nouveau modèle, un associé est responsable uniquement de ses actions dans l'entreprise; autrement dit, il jouit d'une responsabilité limitée. Seuls quelques membres participent directement à la gestion de l'entreprise en tant que directeurs. En théorie, le capital est permanent: au lieu de diviser les biens de la compagnie à la fin de chaque voyage, on emploie les profits au paiement des dépenses de l'année suivante. Ce concept n'est cependant mis en pratique que de façon irrégulière au cours du XVII ${ }^{\mathrm{e}}$ siècle $^{38}$.

La société par actions est particulièrement bien adaptée aux entreprises d'envergure à haut risque comme le commerce outre-mer et les projets de colonisation. Elle permet de rassembler des capitaux importants et de répartir les risques entre de nombreux associés, ce qui allège, pour chaque investisseur, le fardeau lié à l'immobilisation de fonds, pendant plusieurs années, dans le développement des infrastructures ${ }^{39}$. Les risques élevés associés à ces entreprises - et les avantages qu'elles représentent pour l'État, sans engagement financier de sa part - amènent les gouvernements anglais et néerlandais à accorder des privilèges et des pouvoirs considérables aux compagnies qui s'en chargent. Parmi ces privilèges et pouvoirs, on retrouve des droits de commerce exclusifs dans la région d'activité de ces compagnies, des concessions de terres, le pouvoir de nommer des

36. K. G. Davies, The Royal African Company (Londres, Longmans, 1957), 27; William Robert Scott, The Constitution and Finance of English, Scottish and Irish Joint-Stock Companies to 1720 (Gloucester, P. Smith, [1912] 1968), $1: 153$.

37. Henri Lévy-Bruhl, Histoire juridique des sociétés de commerce en France aux XVII et XVIII siècles (Paris, Domat-Montchrestien, 1938), 136-138, 255.

38. Par exemple, durant sa première décennie, la Compagnie anglaise des Indes orientales alterne entre le capital continu et un fonds séparé pour chaque voyage. W. R. Scott, Constitution and Finance..., op. cit., $1: 154-57$.

39. K. G. Davies, The Royal African Company..., op. cit., 32-37. 
officiers de justice et la permission d'armer leurs navires et de construire des ouvrages défensifs dans les colonies ${ }^{40}$. Les gouvernements voient toutes ces concessions, précisées dans une charte, comme de très bons investissements, car ils croient que le meilleur moyen de contrôler les mers est d'établir des routes de commerce maritime, des infrastructures et des forces navales.

\section{LA NOUVELLE-FRANCE SOUS L'AUTORITÉ DE L’AMIRAUTÉ DE FRANCE}

Au début des années 1620, avec la nomination d'un nouveau vice-roi, la Nouvelle-France est pour la première fois placée sous l'autorité d'une institution métropolitaine déjà existante. Ayant acheté le titre de vice-roi du prince de Condé en novembre 1619, Henri de Montmorency, amiral de France, reçoit une commission du roi ${ }^{41}$. Ses lettres patentes n'existent plus, mais il semble qu'elles aient été identiques à celles de Condé, lui conférant l'autorité gouvernementale sur la vallée du Saint-Laurent et l'Acadie ainsi que des droits de traite exclusifs de Matane à Québec et au-delà. Le contexte de ce changement démontre qu'il s'agit à la fois d'une tentative de l'amiral de renforcer son emprise - et, par extension, celle de la Couronne - sur les affaires maritimes, et d'une stratégie dynastique de la part d'une alliance de familles nobles.

Depuis 1582, l'amiral de France revendique le contrôle des ports, des fortifications, des navires, des munitions et de la justice maritime en Picardie, en Normandie, au Poitou, en Saintonge, en Guyenne et en Bretagne. En pratique, son autorité n'est reconnue que dans les deux premières provinces; ailleurs, une importante résistance se manifeste, surtout à La Rochelle qui, malgré la présence d'agents de l'amiral, crée sa propre amirauté au besoin. En Provence, en Languedoc, et dans plusieurs seigneuries, dont celles des Sables-d'Olonne et de Saint-Valéry, l'amiral n'exerce aucune autorité officielle ${ }^{42}$. Même si son autorité administrative n'est pas toujours reconnue, l'amiral jouit d'un important pouvoir personnel grâce à son droit de prise et à ses «droits d'ancrage et du guet». Huit ans avant de devenir

40. Le roi, ou les états généraux aux Pays-Bas, garde sa suzeraineté sur les territoires accordés aux compagnies. E. L. J Coornaert, «European Economic Institutions and the New World; the Chartered Companies ", dans The Cambridge Economic History of Europe (Cambridge, Cambridge University Press, 1966), $4: 245-250$.

41. Même si elle a fait l'objet d'une commission du roi, la vice-royauté devient vénale lorsque Montmorency l'achète de Condé pour 30000 livres. Voir Roland Mousnier, Les institutions de la France sous la monarchie absolue, 1598-1789 (Paris, Presses universitaires de France, 1980), 2: 457.

42. A. James, Navy and Government..., op. cit., 34-36; David Parker, La Rochelle and the French Monarchy: Conflict and Order in Seventeenth-Century France (Londres, Royal Historical Society, 1980), 74-76. 
vice-roi de Nouvelle-France, Henri de Montmorency succède à son oncle, Charles de Montmorency, comme amiral. Comme son prédécesseur, Henri travaille à la centralisation de son autorité par une combinaison de pouvoirs personnels et administratifs, avec l'approbation du roi. Il tente de renforcer les sièges particuliers de l'amirauté et il envoie ses agents personnels dans les ports pour percevoir les droits qui lui sont dus en vertu des procédures de ces amirautés ${ }^{43}$. En 1622, la Couronne augmente l'autorité des amirautés, de l'amiral lui-même et du conseil royal, puis, quatre ans plus tard, un poste de secrétaire est créé pour chacune des marines, celle du Ponant et celle du Levant. Montmorency achète également le titre d'amiral de Guyenne d'Henri de Châtillon, comte de Coligny, dont la famille est apparentée à celle de l'amiral par l'arrière-grand-mère de ce dernier.

C'est dans ce contexte qu'il faut voir l'achat de la vice-royauté de Nouvelle-France. Comme l'acquisition de l'amirauté de Guyenne, l'obtention de cette charge outre-mer renforce le pouvoir de Montmorency comme amiral. Les deux transactions permettent aux familles aristocratiques de distribuer des intérêts maritimes pour des raisons stratégiques; dans ce cas, Condé était le beau-frère de Montmorency. De façon plus significative, le prince, en tant que vice-roi, a le droit de délivrer des licences en France aux commerçants partant pour le Saint-Laurent, une prérogative qui est théoriquement en compétition avec les pouvoirs de l'amiral sur tous les navires qui entrent ou sortent des ports - si elle ne sape pas ces pouvoirs. Sur la recommandation de l'intendant de l'amirauté, le sieur de Villemenon, Montmorency ajoute la vice-royauté à son domaine personnel et institutionnel ${ }^{44}$. Il n'est probablement pas au courant de la situation de la colonie: un petit rassemblement de colons, presque sans infrastructure, dans un territoire sous le contrôle de peuples autochtones. Par contre, la Nouvelle-France intéresse l'amiral en tant que zone maritime. Son autorité sur la colonie, comme sur n’importe quel port français, étend la portée de sa surveillance de la circulation des biens et des sujets. Pour la première fois, le contrôle du trafic maritime en France est lié au contrôle de territoires outre-mer.

La vice-royauté de Nouvelle-France n'est pas simplement liée à l'amirauté par la personne de Montmorency, elle profite de l'expertise admi-

43. A. James, Navy and Government..., op. cit., 58; voir aussi 24, 40, 50, 62, 76.

44. D. Parker, La Rochelle and the French Monarchy..., op. cit., 75-76; L.-A. Boiteux, Richelieu, grand maître..., op. cit., 67 ; D. H. Fischer, Le rêve de Champlain..., op. cit., 423-426; M. Trudel, HNF, op. cit., 2 : 264-265. Sur les différentes licences délivrées par Condé en tant que vice-roi, voir Émile Gosselin, Nouvelles Glanes historiques normandes (Rouen, 1873), 34, 38-39. 
nistrative et maritime de cette institution, à travers une configuration semblable d'agents personnels et de postes administratifs, tels que celui d'intendant. Montmorency désigne à son tour Champlain comme son lieutenant et lui confère le commandement de Québec, fonctions presque identiques à celles que Champlain avait obtenues sous Condé. Pour la première fois de l'histoire de la colonie, il y a aussi un intendant de la Nouvelle-France, Jean-Jacques Dolu, conseiller du roi et grand audiencier de France. Les responsabilités de Dolu sont définies de façon très large, englobant les "choses concernant les affaires de la Nouvelle-France», y compris le pouvoir de conclure des contrats avec des marchands pour les provisions et marchandises et même de déterminer si la Compagnie de Rouen et Saint Malo devrait conserver ses droits de commerce dans la colonie $^{45}$. Champlain applaudit à la nomination de Dolu, en espérant qu'elle «[mettra] nostre Societé en meilleur estat de bien faire qu'elle n'[a] fait ${ }^{46} »$. Montmorency nomme également deux commissaires, Baptiste Guers et le capitaine du May, qui font le voyage en Nouvelle-France en 1620 afin d'en ramener un rapport sur l'état de la colonie. Guers, du moins, fera d'autres voyages aux mêmes fins, semble-t-il, et agira comme messager, livrant à Champlain des lettres du roi, de Montmorency, de Dolu, des associés de la compagnie qui obtient des droits de commerce en 1620 et du Sieur de Villemenon. Ce dernier correspondant ne possède aucune charge particulière concernant la Nouvelle-France, mais il agit par moments comme intermédiaire entre Montmorency et la compagnie ${ }^{47}$. L'étendue réelle des fonctions confiées à Dolu, à Guers et à du May n'est pas connue, mais il est possible et même probable que, tout comme l'intendant de l'amirauté et les commis dans les ports français, ces hommes aient pour rôle d'affirmer et de protéger les droits de Montmorency en tant que vice-roi ${ }^{48}$.

Tout comme les auteurs de mémoires cités plus haut, Montmorency voit les compagnies inspirées des modèles néerlandais et anglais comme un moyen inestimable non seulement de stimuler la navigation et le com-

45. Bibliothèque et Archives Canada (ci-après BAC), Minutier central (ci-après MC), v. L, étude LIV, $\mathrm{n}^{\circ}$ 495, "Procuration de Montmorency à Dolu », 24 février 1620; M. Trudel, HNF..., op. cit., 2: 265-266.

46. H. P. Biggar, dir., The Works of Samuel de Champlain (Toronto, University of Toronto Press, [19221935] 1971), $4: 368$.

47. M. Trudel, HNF..., op. cit., 2: 277-278; D. H. Fischer, Le rêve de Champlain..., op. cit., 423-426.

48. Quand Dolu acquiert une action dans la Compagnie de Montmorency, en novembre 1622, il souligne son statut de sujet ordinaire du roi, ayant déjà démissionné du poste d'intendant. Il ne veut pas être vu comme l'homme de Montmorency dans la compagnie. Cependant, il continue peut-être de lui faire rapport de façon officieuse. Voir BnF, dépt. de ms., Collection Margry, v. 9269, f. 190-191v, 5 novembre 1622 . 
merce français, mais également d'étendre sa propre juridiction aussi loin que les Français voyagent par la mer ${ }^{49}$. En effet, la création d'une compagnie pour la Nouvelle-France lui offre un moyen très pratique de consolider son pouvoir sur la colonie et sur son commerce. Montmorency est en mesure de décider de la composition de la compagnie, de son organisation et de ses conditions de fonctionnement. Contrairement au prince de Condé, il participe déjà à une compagnie au moment où il devient vice-roi de la Nouvelle-France en 1620. Depuis 1615, il parraine officiellement la Compagnie des Indes orientales (CIOr), qui jouit de droits de commerce exclusifs dans les Indes orientales ${ }^{50}$. Même si, sous Montmorency, la Compagnie de la Nouvelle-France émerge de son contexte particulier, l'entreprise asiatique et elle-même ont en commun certaines caractéristiques et, parfois, certains membres de leurs personnels. En 1620, Dolu, au nom du vice-roi, entame des négociations avec Ezechiel de Caen, marchand rouennais et l'un des administrateurs de la CIOr, ainsi que son neveu Guillaume de Caen, capitaine de navire, pour établir ce qui deviendra la Compagnie de Montmorency pour la Nouvelle-France. Même si les de Caen ne sont pas de véritables clients de Montmorency, il est intéressant de noter qu'ils étaient déjà à son service à d'autres titres. Le vice-roi prévoit également faire surveiller étroitement les activités de la Compagnie de Montmorency par ses agents, en particulier par l'intendant de l'amirauté ou par l'intendant de la Nouvelle-France ${ }^{51}$. La disposition concernant cet aspect a pour effet de transférer le siège de l'association depuis les ports où les navires sont équipés vers Paris, base de Montmorency. Afin d'accroître son autorité personnelle et institutionnelle en France, ce dernier s'assure le contrôle d'entreprises commerciales et de colonisation outre-mer.

49. Toutefois, Montmorency les approche avec une plus grande prudence que certains de ses contemporains. Étant donné l'envergure des entreprises outre-mer et les risques qui y sont associés, il recommande une période probatoire de deux ou trois ans avant d'émettre des lettres patentes officielles. Il n'y a aucune indication que cette pratique ait existé. Voir L.-A. Boiteux, Richelieu, grand maître, op. cit., 79-80.

50. Sur l'histoire de la compagnie, voir «Lettres patentes par Louis XIII pour la première Compagnie des Indes Orientales ", 2 juillet 1615, dans Jacques du Fresne de Francheville, Histoire de la Compagnie des Indes (Paris, 1746), 162-166; Philippe Haudrère, La Compagnie française des Indes au XVIII siècle (Paris, Indes savantes, 2005), 1: 19-23 ; A. James, The Navy and Government in Early Modern France..., op. cit., 41-42.

51. Robert Le Blant et René Baudry, dir., Nouveaux documents sur Champlain et son époque (ci-après Nouv. docs.), 1: 408. Quand la CNF est créée en 1627, son intendant préside également les assemblées générales de la compagnie. Voir plus bas. 


\section{RICHELIEU VISE LE CONTRÔLE MARITIME}

\section{«Les compagnies universelles »}

La consolidation de l'autorité maritime de Montmorency provoque des contre-attaques de la part de ses rivaux personnels et institutionnels. Vers le milieu des années 1620, le cardinal de Richelieu, l'un des principaux rivaux de Montmorency en matière d'influence auprès du roi et, à titre de ministre, principal défenseur des intérêts du roi, commence à s'intéresser aux affaires maritimes pour des raisons personnelles et stratégiques. À ce moment, Montmorency exerce déjà une forte emprise féodale sur l'amirauté: les postes clés sont occupés par des hommes qui affichent une loyauté inébranlable envers lui et qui porteraient des armes pour lui comme tout vassal à l'égard de son seigneur. Réalisée grâce à la présence d'agents dans les ports clés, à l'obtention de postes de gouverneur, à la mobilisation d'un vaste réseau clientéliste et à une vigoureuse chasse aux compétiteurs, cette base politique menace de saper les intérêts de la Couronne ainsi que l'autorité et les ambitions personnelles de Richelieu dans une période où le contrôle de la mer devient particulièrement important.

En 1626, des événements nationaux et internationaux ont déterminé la Couronne à manifester plus activement son intérêt pour le commerce maritime et la navigation. Bien que religieux au début, les troubles huguenots des trois premières décennies du XVII ${ }^{\mathrm{e}}$ siècle traduisent bien plus que des différences confessionnelles. Les huguenots veulent en effet maintenir sous bonne garde leur indépendance commerciale et politique. Un élément clé des guerres de Religion est donc le contrôle de la mer ${ }^{52}$. La Rochelle, centre du pouvoir huguenot, possède des navires bien armés qui contrôlent en pratique la côte atlantique, délivre des licences sans consulter les lieutenants de l'amiral et héberge divers marchands actifs dans le commerce outre-mer. En réalité, ce port est un symbole de l'impuissance de la marine royale et de son manque de contrôle du commerce maritime. Des conflits répétés avec les huguenots le long de la côte atlantique, dans les années 1610 et 1620, fournissent des preuves concrètes de cette vulnérabilité. Non seulement les forces royales sont défaites à plusieurs reprises par des sujets rebelles et dépendent de l'aide de marines étrangères pour réagir, mais la France doit également faire face à la menace d'une invasion de la part de la redoutable marine anglaise, prête à soutenir ses coreligionnaires. Le cardinal de Richelieu soupçonne les Anglais - qui se méfient

52. Sur l'argument voulant que le siège de La Rochelle traduit l'engagement personnel de Louis XIII envers la "conformité religieuse», voir A. James, The origins of French absolutism..., op. cit., 27-28. 
des efforts de la France pour augmenter sa puissance maritime - de ne pas entendre se limiter à défendre La Rochelle et les îles extérieures, mais de vouloir profiter de l'occasion pour mettre la main sur des ressources précieuses, comme le sel, dans les provinces avoisinantes. En juillet 1627, une flotte anglaise attaque les défenses royales sur l'île de Ré, un emplacement stratégique clé au large de la côte atlantique. C’est avec grande difficulté et en faisant appel à différents ports, que Richelieu parvient à rassembler à la hâte une force navale pour repousser les Anglais, ce qui permet à l'armée française de se concentrer sur le siège de La Rochelle, dernier bastion des huguenots. En octobre 1628, lorsque le port capitule, les huguenots, en tant que force politique et militaire, sont épuisés ${ }^{53}$.

Ce défi lancé de l'intérieur au pouvoir commercial et naval du roi trouve son corollaire international durant la même décennie, alors que bon nombre des pays d'Europe s'emploient à renforcer leur marine en prévision d'une éventuelle recrudescence des conflits navals et pour protéger leur commerce maritime. En 1618, l'Angleterre entreprend de construire une flotte de navires de guerre dans les cinq années suivantes. Au début des années 1620, l'Espagne met en vigueur la Pragmática, qui interdit l'importation de tout produit manufacturé à l'étranger non absolument nécessaire au commerce intérieur ou international, et l'Almirantazgo, ou l'amirauté de Séville, qui réglemente et protège le commerce maritime. Les Espagnols lancent également l'Armada de Flandre, une force navale traditionnelle, et vont jusqu'à inspecter des navires dans les eaux internationales et à appuyer les corsaires ${ }^{54}$. La France s'inquiète particulièrement de l'augmentation combinée des forces navales et des limitations au commerce. Ces développements sur les scènes internationale et intérieure convainquent Louis XIII et Richelieu que la pratique traditionnelle de la Couronne, soit de confier à des individus des activités fondamentales pour

53. D. Parker, La Rochelle and the French Monarchy..., op. cit., 71-82; Mack P. Holt, The French wars of religion, 1562-1629 (Cambridge, Cambridge University Press, 2005), 190-191, deuxième édition; Richard Bonney, Political Change in France Under Richelieu and Mazarin, 1624-1661 (Oxford, Oxford University Press, 1978), 387-389.

54. Henri Hauser, La Pensée et l'action économiques du Cardinal de Richelieu (Paris, Presses universitaires de France, 1944), 54-56; V.-L. Tapié, France in the Age of Louis XIII and Richelieu..., op. cit., 141-142; R. Briggs, "Richelieu and Reform... », loc. cit., 86; A. James, Navy and Government..., op. cit., 43-46. Les historiens ne sont pas unanimes concernant l'importance relative que Richelieu donne aux menaces anglaise et espagnole. Sur la menace anglaise, voir A. James; pour la menace française, Erik Thomson, «France’s Grotian Moment? Hugo Grotius and Cardinal Richelieu’s Commercial Statecraft ", French History, 21,4 (2007): 378 . 
la réputation et la sécurité de la France, ne suffit plus à protéger les intérêts royaux ${ }^{55}$.

C'est dans ce contexte que Richelieu commence à se faire attribuer personnellement l'autorité maritime militaire et commerciale. Aux yeux du cardinal, l'amirauté représente un obstacle majeur à la mission de la Couronne d'endosser la responsabilité de la sécurité maritime. À travers la création de compagnies, Richelieu cherche simultanément à rétablir la marine et le commerce français et à étendre son pouvoir personnel sur les affaires maritimes aux dépens de l'amiral. Le 31 mars 1626, il conclut un contrat notarié en son nom avec Guillaume de Bruc, Jean-Baptiste du Val, Nicolas Le Mareschal et Antoine Regnault de Montmort afin d'établir la Compagnie du Morbihan. Celle-ci a pour mission "[le] commerce general en touttes sortes de marchandizes manufacturées et autres choses possible et licites... tant par mer que par terre, levant, ponant et voyages de long cours... partout tant dedans que hors le royaume ${ }^{56}$ ”.

Contrairement aux autres compagnies maritimes, elle n'est pas sous l'autorité de l'amiral de Montmorency et elle n'a pas à payer les obligations coutumières ni à obtenir de congés. Au contraire, elle relève du «surintendant général du commerce», soit le cardinal lui-même. Tentative délibérée de saper l'autorité de l'amiral, ce contrat met tout, sauf la réglementation des pêches, sous la responsabilité de Richelieu ${ }^{57}$. Le domaine des pêches ainsi que le contrôle de toute manufacture nouvelle ou existante, est, depuis mai, sous le contrôle de la Compagnie de la Nacelle de Saint-Pierre fleurdelisée. Comme dans le contrat de la première compagnie, Richelieu s'y engage, à titre de surintendant, à garder les associés de la compagnie "par devant soi et leur en donnera l'autorisation necessaire

55. L.-A. Boiteux, Richelieu, grand maître..., op. cit., 49-51 ; P. Castagnos, Richelieu face à la mer..., op. cit., 97. Cette prise de conscience se traduira de nouveau par la réunion de la marine et du commerce maritime sous la responsabilité d'une seule personne. Cette fois, il s'agit de Richelieu. Même si l'autorité personnelle de ce dernier et celle de Louis XIII ne sont pas interchangeables, le fait que le cardinal prolonge l'autorité royale au moyen de ses réseaux clientélistes et familial fait en sorte que, bien des fois, les mêmes actions font avancer et les objectifs royaux et les intérêts personnels de Richelieu. Par exemple, l'acquisition par Richelieu du poste de gouverneur de la Bretagne renforce le droit de regard de la Couronne sur les affaires maritimes tout en consolidant l'empire personnel de Richelieu. Voir A. James, The origins of French absolutism..., op. cit., 60-63, 73-74.

56. "Contrat de la Compagnie du Morbihan, 31 mars 1626 ", dans Papiers de Richelieu..., op. cit., 1 : 305 ; L.-A. Boiteux, Richelieu, grand maître..., op. cit., 87.

57. BAC, C11A, correspondance générale (ci-après corresp. gén.), «Edit du roi pour l'établissement de la Compagnie des Cent-Associés ", juillet 1626; L.-A. Boiteux, Richelieu, grand maître..., 90-91. Un édit émis au mois d'août va plus loin en supprimant l'amirauté de France, Bretagne et Guyenne et en en cédant l'autorité au «surintendant du commerce». Voir Le Réseau du Canada..., op. cit., 50, 53-54. 
pour en faciliter l'execution ${ }^{58}$ ». Le roi réserve aux deux compagnies un ou deux ports en France pour qu'elles y établissent leurs sièges, et leur délègue en même temps ses pouvoirs de justice, d'administration et d'imposition.

L'autorité des deux compagnies s'étend jusqu'en Nouvelle-France, même si leurs contrats respectifs n'en définissent pas précisément les frontières. L'ambiguité autour des limites exactes de la colonie met en relief les hautes aspirations associées à ces compagnies. Sans beaucoup d'égard à la réalité coloniale, on s'intéresse surtout à ce que représentent la Nouvelle-France et les compagnies: une façon d'améliorer la réputation de la France, une source de profits futurs par le commerce et un moyen d'étendre l'autorité sur les sujets qui voyagent à l'extérieur de la métropole. La Compagnie du Morbihan peut «jouir et posséder les terres de la Nouvelle France tant le continant qu'isles et autres lieux, que ladite compagnie pourra conquérir et peupler", tandis qu'on demande à la Compagnie de la Nacelle d'établir des colonies partout où cela est approprié, y compris en Nouvelle-France, et de conquérir de nouvelles terres qui ne sont pas encore sous l'autorité du roi ${ }^{59}$. La Couronne ne se réserve pas «[d']autre droit sur lesdits terres et pays que celui de la souveraineté foi et hommage» et le cadeau d'une couronne en or lors de la succession de chaque nouveau roi ${ }^{60}$. Il semble que les deux compagnies se voient accorder explicitement «la pleine et entière possession, jouissance, et disposition» des terres qu'elles découvrent ou qu'elles conquièrent, uniquement, et non des terres déjà possédées par la France ${ }^{61}$. Connues comme des "compagnies universelles», les Compagnies du Morbihan et de la Nacelle dépassent de loin les paramètres des compagnies étrangères qui les ont inspirées, tant sur le plan géographique qu’à l'égard de la gamme d'activités qu'elles englobent.

58. BAC, Collection Dupuy, v. 318, «Contrat fait par Monsieur Le Cardinal de Richelieu... avec une compagnie de Flamens [sic] et autres associés», 19 mai 1626.

59. Il convient de signaler que l'édit qui crée officiellement la CNF ne contient pas les mots «conquérir» ou "conquête». Il est difficile de déterminer pourquoi ils n’y paraissent pas, mais on peut penser que le mandat premier de la compagnie, l'évangélisation, et son rôle possible dans la pacification d'une faction mécontente et ardemment catholique, le parti dévot, qui s'oppose à la guerre avec l'Espagne et aux alliances avec des pays huguenots, fournit une explication au moins partielle. Les commissions accordées aux gouverneurs et lieutenants-généraux de Nouvelle-France après 1627 continuent de comprendre ces mots. Voir, par exemple, la commission de Charles Menou, sieur d'Aulnay, qui date de février 1647 dans Collection de manuscrits contenant lettres, mémoires, et autres documents historiques relatifs à la Nouvelle-France (Québec, 1884), 123.

60. BAC, C11A, corresp. gén., "Edit du roi pour l'établissement de la Compagnie des Cent-Associés», juillet 1626. Voir aussi BAC, Collection Dupuy, v. 318, "Contrat fait par Monsieur Le Cardinal... », op. cit.

61. BAC, Collection Dupuy, v. 318, "Contrat fait par Monsieur Le Cardinal... », op. cit. 
Sur les plans politique et logistique, cependant, il s'avère très difficile, voire impossible de mettre sur pied des entreprises aussi vastes, comme le démontrent bientôt les événements. Leurs mandats très larges, qui englobent tout commerce intérieur et international, attirent une opposition véhémente de la part de différents secteurs économiques et de différentes régions du pays, mais surtout en Bretagne, site proposé pour l'établissement de la Compagnie du Morbihan. Réagissant à la fois aux privilèges commerciaux exclusifs attribués à la compagnie et à sa tentative de saper le pouvoir du gouverneur provincial, dont l'autorité englobait traditionnellement l'amirauté, le parlement de Rennes refuse en fin de compte d'enregistrer l'édit. Et même si les États provinciaux le font, c'est avec des conditions si sévères que celles-ci neutralisent l'objectif de départ ${ }^{62}$. Ces contretemps dans l'enregistrement de la compagnie, conjugués à la difficulté de trouver le nombre d'associés nécessaire, font comprendre à Richelieu que ces entreprises ne faciliteront ni son accession à un nouveau titre et à de nouveaux pouvoirs ni l'accroissement de la puissance maritime française.

\section{«La Grande Maîtrise »}

La Couronne finira par reprendre le contrôle des affaires maritimes au moyen d'une fonction qui ressemble beaucoup à celle d'amiral, bien qu'elle n'en porte pas le nom. Ainsi, les objectifs conjugués du rétablissement du commerce et de la marine et de la réduction de l'amirauté reçoivent l'approbation du roi, en octobre 1626, lorsque Richelieu reçoit des lettres patentes le nommant "Grand Maitre, Chef et surintendant général de la navigation et du commerce de France $^{63}{ }^{\prime}$. Ces lettres patentes lui accordent les responsabilités traditionnelles de l'amiral: assurer «la seureté de noz subiectz en la mer, portz, havres, rades et grèves d'icelle et isles adjacentes» et l'observance des ordonnances marines, éliminer les corsaires et les pirates et réunir une flotte capable de protéger les côtes et le

62. Les États enregistrent l'édit en janvier 1628. G. Carpin, Le Réseau du Canada..., op. cit., 55 ; Kenneth M. Dunkley, "Trading Companies and Privilege in Richelieu's France: The Hundred Associates of Morbihan", Proceedings of the French Colonial Historical Society, 3 (1977): 5-9. L'opposition se manifeste également contre la Compagnie de la Nacelle, en particulier contre sa proposition de recruter des familles flamandes pour développer l'industrie et le commerce français. Voir BnF, dépt. de ms., fonds français, v. 4826, "Discours sur la verification du Contract Concernant le commerce et traficq», c.1627; G. Carpin, Le Réseau du Canada..., op. cit., 57.

63. Même si la Compagnie du Morbihan est un peu en perte de vitesse quand Richelieu obtient ses propres lettres patentes, elle n'est abandonnée de façon définitive qu'en 1628. Voir G. Carpin, Le réseau du Canada..., op. cit., 55 n. 39, 58 n. 55 ; L.-A. Boiteux, Richelieu, grand maître, 100-101. La Compagnie de la Nacelle subsiste au moins jusqu'en mars 1627, quand Richelieu prépare l'édit de la compagnie afin de le présenter de nouveau aux parlements. Voir Papiers de Richelieu..., op. cit., 2: 124-125. 
commerce océanique en temps de paix. Les responsabilités liées à cette charge indiquent également la méthode par laquelle la puissance navale et le commerce maritime français seraient assurés: «traicter avec touttes sortes de personnes, voir et examiner les propositions qui nous ont este et seront faictes sur le sujet de l'establissement du commerce et discuter et recongnoistre le merite[,] bien et utilité, resoudre et asseurer tous articles, traictez, contractz et conventions avec tous ceux qui se voudront lier et joindre pour former lesdictes sociétés et compagnies du commerce ${ }^{64}$.» Si Richelieu a bon espoir que ces lettres patentes seront approuvées plus facilement que celles des compagnies universelles, il comprendra rapidement son erreur. En effet, bien que le parlement de Rennes enregistre les lettres patentes en avril, il refuse au titulaire le pouvoir de "faire armement de guerre", ne reconnaissant que les pouvoirs de délivrer des licences pour des voyages océaniques et de surveiller les gardes côtières ${ }^{65}$. Étant donné les difficultés rencontrées lors de l'enregistrement, il n'est pas surprenant que la mise en vigueur des lettres patentes s'avère un défi considérable.

Le titre pompeux de la charge cache mal le fait que le cardinal met la main sur l'amirauté aux dépens de Montmorency. En effet, Richelieu adopte plusieurs des mesures utilisées par son prédécesseur pour affirmer son autorité administrative et ses privilèges personnels. Une des premières choses qu'il fait est d'envoyer des agents maritimes aux ports du royaume pour affirmer ses droits et faire rapport sur l'état des infrastructures côtières, tâches auparavant accomplies par les agents de Montmorency ${ }^{66}$. Au sein de son conseil de la marine, Richelieu élargit le mandat du conseil des prises de Montmorency, qui porte sur les prises saisies en mer, pour y ajouter le pouvoir d'enquêter sur des allégations d'abus de la part d'agents de l'amirauté et d'évaluer des propositions commerciales. Sous

64. BnF, dept. de ms., Cinq Cents de Colbert, v. 203, «Lettres patentes de création de la charge de Grand Maître Chef et surintendant général de la navigation et commerce de France», f. 203. Dans son argument selon lequel l'autorité navale importe par-dessus tout, James se désintéresse complètement de la perception du rôle des compagnies dans cette même autorité et du nombre de compagnies proposées et acceptées durant la période, qu'elles soient privées ou sous la supervision de Richelieu. Voir A. James, Navy and Government..., op. cit., 53-54.

65. L.-A. Boiteux, Richelieu, grand maître..., op. cit., 103. Les lettres avaient été enregistrées par les parlements de Paris et de Rouen le 18 mars et le 16 avril, respectivement. Le parlement de Bordeaux ne les enregistre pas avant la fin mai. Voir G. Carpin, Le Réseau du Canada..., op. cit., 72.

66. Pour des exemples de rapports soumis à Richelieu, voir «Voyage et inspection maritime de M. D’Infreville», dans Eugène Sue, Correspondance de Henri D'Escoubleau de Sourdis augmentée des ordres, instructions et lettres de Louis XIII et du Cardinal de Richelieu, (Paris, L'imprimerie de Crapelet, 1839), 3 : 214 ; BnF, dépt. de ms., fonds français, v. 18596, «Rapport de Nicolas Langlois sieur du Collemoulins... sur les ports et havres de Normandie », 22 mars 1627; A. James, Navy and Government..., op. cit., 62, 69-70. 
Montmorency, l'intendant Villemenon assurait la reconnaissance des privilèges de l'amiral par les gouverneurs provinciaux et les seigneurs, deux groupes qui revendiquaient souvent l'autorité judiciaire et des droits d'amirauté. Sous Richelieu, l'homologue de Villemenon est d'abord Martin de Mauvoy, puis le marquis d'Effiat. En général, le cardinal maintient et développe l'administration mise en place sous Montmorency, au lieu de se lancer dans une nouvelle direction ${ }^{67}$.

Contrairement à l'argument voulant que Richelieu ait établi un véritable ministère de la Marine, il faut reconnaître que sa capacité de bien exercer sa charge, comme dans le cas des amiraux qui l’ont précédé, dépend en grande partie de son autorité personnelle ${ }^{68}$. Il affecte des parents et des clients à des postes administratifs, comme son oncle, Amador de la Porte, chef des escadres et intendant général de la marine et du commerce. Par la suite, il étend son emprise sur le Levant en achetant le titre de général des galères et il conteste, devant le conseil du roi, les droits d'amirauté des seigneurs des Sables-d'Olonne, de Bayonne et d'ailleurs ${ }^{69}$. Dès le début, il obtient les postes de gouverneur dans des ports clés, dont Le Havre, Brest et Brouage, pour lui-même ou pour ses clients. Même s'il jouit d'un accès privilégié à Louis XIII, à titre de ministre principal, l'exercice réel du pouvoir de Richelieu passe par ces réseaux de soutien et de fidélité. Cette méthode souligne l'importance d'un aspect clé du pouvoir politique au début du XVII ${ }^{e}$ siècle: de nature très féodale, il repose sur la fidélité et l'intérêt personnel; les responsabilités officielles et les émoluments individuels allaient de pair. En tant que grand maître, Richelieu pouvait tout à la fois étendre le contrôle royal sur des charges clés, dans le domaine de la navigation, et consolider ses intérêts patrimoniaux ${ }^{70}$.

\section{COMBAT TRANSATLANTIQUE: UNE NOUVELLE FORME D'ADMINISTRATION POUR LA NOUVELLE-FRANCE?}

Le cardinal, tout comme Montmorency, voit son pouvoir sur les mers comme ayant la même étendue que les entreprises de colonisation et de commerce françaises et, par extension, la Nouvelle-France. L'un et l'autre

67. A. James, Navy and Government, op. cit, 57-59, 62 ; L.-A. Boiteux, Richelieu, grand maître..., op. cit., 114-117.

68. Sur la grande maîtrise vue comme un ministère de la Marine, du Commerce, et des Colonies, voir L.-A. Boiteux, Richelieu, grand maître..., op. cit., 99-100.

69. L.-A. Boiteux, Richelieu, grand maître..., op. cit., 115-116, 133, 150-151; A. James, Navy and Government..., op. cit., 65 ; P. Castagnos, Richelieu face à la mer..., op. cit., 68-70.

70. D. Parker, “Class, Clientage, and Personal Rule... », loc. cit., 206; W. Beik, Absolutism and Society..., op. cit., chap. 9 , en particulier la partie 4 . 
établissent un lien entre l'édification de l'État en France et la souveraineté dans les colonies. La Nouvelle-France est un élément stratégique de ce processus, élément que l'on peut maîtriser au moyen des compagnies commerciales et de colonisation.

Voir la Nouvelle-France dans ce contexte transatlantique transforme notre compréhension des circonstances qui ont présidé à l'établissement de la Compagnie de la Nouvelle-France et souligne certaines caractéristiques de la formation de l'État durant cette période. Pendant que les deux compagnies universelles sont toujours devant les parlements, Richelieu passe un troisième contrat pour instituer la CNF. En avril 1627, cette nouvelle association reçoit «en toute propriété, justice et seigneurie»le fort et l'habitation de Québec et «tout le dit pays de la Nouvelle-France [...] tant le long des côtes depuis la Floride [...] jusqu'au cercle Arctique pour latitude, et de longitude depuis l'Isle de Terre Neuve, tirant à l'ouest, jusqu'au grand lac, dit la mer douce, et au-dela» en échange des actes habituels d'allégeance à la Couronne ${ }^{71}$. Le conflit apparent avec la compétence des deux compagnies universelles a amené certains spécialistes à affirmer que la CNF avait émergé des cendres de ces deux entreprises. Plus d'un auteur a affirmé que la Compagnie du Morbihan avait précédé la Compagnie de la Nacelle et qu'elle constituait l'antécédent de la Compagnie de la Nouvelle-France ${ }^{72}$.

Cependant, des recherches récentes démontrent que toutes trois furent mises en œuvre de façon quasi simultanée: des associés de la Compagnie du Morbihan s'engagent avec Richelieu en mars 1626, ceux de la Compagnie de la Nacelle, en mai de la même année, et ceux de la CNF en mars de l'année suivante ${ }^{73}$. Même si le chevauchement des compétences témoigne peut-être du désir du cardinal de s'assurer que la Nouvelle-France relève bien d'une compagnie, en fin de compte, de tels chevauchements sont très fréquents dans les contrats liés à des entreprises en milieu colonial ${ }^{74}$. Je soutiens que l'importance de ces parallèles et de ces chevauchements réside

71. BAC, C11A, corresp. gén., «La Compagnie de Canada, et sous le titre de la Nouvelle France, par les articles des 29 avril et 7 mai, 1627», f. 79-84.

72. P. Castagnos, Richelieu face à la mer..., op. cit., 73 ; L.-A. Boiteux, Richelieu, grand maître..., op. cit., 243 ; Pierre Bonnassieux, Les grandes compagnies de commerce (New York, Burt Franklin, [1892] 1969), 358360, 363. La confusion entre les Compagnies du Morbihan et de la Nouvelle-France s'explique en partie par le fait qu’elles partagent le même surnom: «les Cent-Associés». Thomson perpétue cette confusion dans «France’s Grotian Moment?», op. cit., 389.

73. G. Carpin, Le réseau du Canada..., op. cit., 58; L.-A. Boiteux, Richelieu, grand maître..., op. cit., 235.

74. Christopher Tomlins, «Law's Empire: Chartering English Colonies on the American Mainland in the Seventeenth Century", dans Diane Kirkby et Catharine Coleborne, dir., Law, History, Colonialism: The Reach of Empire (Manchester, Manchester University Press, 2001), 30. 
ailleurs; n'ayant aucune politique unique sur les entreprises commerciales et de colonisation, le roi et Richelieu font des essais et approuvent des initiatives pour diverses raisons: le désir de confier tout le commerce français à quelques grandes compagnies (les compagnies universelles), le besoin de régler des problèmes précis dans des colonies existantes (la CNF), le désir d'appuyer des entreprises privées dont les intérêts correspondent à ceux de la Couronne (la Compagnie de St-Christophe, dans les Indes occidentales, établie en 1626) et, la dernière et non la moindre, l'ambition politique de Richelieu lui-même. Les contingences, le manque de cohérence et la contestation marquent les processus jumelés de la formation de l'État et l'extension de la souveraineté au domaine colonial.

La CNF combine de nouvelles formes d'organisation et des méthodes traditionnelles d'exercice du pouvoir, particularité que masque sa réputation de première grande compagnie d'outre-mer à atteindre une certaine viabilité. L'historiographie de la Nouvelle-France présente traditionnellement cette compagnie comme un nouveau départ dans l'organisation administrative et commerciale de la colonie. À première vue, les différences sont assez frappantes: pour la première fois dans l'histoire de la Nouvelle-France, aucun titre n'est accordé à un individu qui aurait le pouvoir administratif et les privilèges de traite; c'est plutôt à une association que revient le titre. Ainsi, le contrat confère à la $\mathrm{CNF}$ des pouvoirs royaux, y compris la défense, la production de canons et de munitions, la distribution de terres et la désignation de capitaines. Même si on prévoit que certaines décisions seront prises collectivement par les associés, la majorité des affaires sont traitées par douze directeurs élus, comme dans les deux compagnies universelles et suivant les exemples néerlandais et anglais. Un examen plus attentif révèle cependant une grande continuité avec la période précédente en ce qui concerne la structure et l'exercice de l'autorité dans la colonie.

Malgré l'édit royal qui confère à la compagnie des pouvoirs gouvernementaux, Richelieu est, dans les faits, le vice-roi de la Nouvelle-France, même s'il n'en porte pas le titre. Le 29 juin, Jean de Lauson, allié de Richelieu récemment nommé intendant de la CNF et de la NouvelleFrance, achète la vice-royauté et les actions qui s'y rapportent dans la Compagnie de Montmorency du vice-roi d’alors, Henri de Lévis, duc de Ventadour. Il paie 72000 livres, que le duc reçoit sous forme de trois charges officielles ${ }^{75}$. Une lettre envoyée par Lauson à Richelieu le lende-

75. BAC, MC, v. L, étude LI, n. 86, «Vente par le duc de Ventadour à Jean de Lauson de la charge de vice-roy au Canada », 29 juin 1627. 
main, pour annoncer la transaction réussie, démontre qu'il agissait sur instruction de son patron ${ }^{76}$. Depuis 1625 , Ventadour exerçait son autorité sur l'administration de la Nouvelle-France par l'entremise de son lieutenant, Samuel de Champlain, et jouissait des privilèges de traite attribués à la compagnie de Montmorency. Comme Henri de Montmorency et les seigneurs qui prétendaient avoir des droits d'amirauté, la situation de Ventadour présente un obstacle à l'objectif de Richelieu de centraliser le pouvoir maritime. Même si cet achat semble être fait au nom de la compagnie, tout porte à croire qu'il est probablement conclu pour le compte du cardinal et qu'il constitue un élément de la consolidation de l'autorité du grand maître ${ }^{77}$. Comme le laissait prévoir la création des compagnies du Morbihan et de la Nacelle, la Nouvelle-France est maintenant sous l'autorité du cardinal.

Si la discrétion avec laquelle la vice-royauté change de mains donne à penser que Richelieu préfère voir la Nouvelle-France relever, en fait, d'une compagnie, sa relation plutôt vice-royale avec la CNF est évidente dans l'administration de la compagnie. À l'instar de Montmorency, il rétablit le poste d'intendant en y nommant Jean de Lauson ${ }^{78}$. Comme dans le cas de Dolu avant lui, les responsabilités de Lauson comprennent la tenue des assemblées générales annuelles de la CNF. Richelieu nomme également des commandants de forts et des capitaines de navires royaux parmi les associés, sous la recommandation de la compagnie ${ }^{79}$. Et, comme les vicerois, il est un associé, autorise les voyages de la compagnie et s'attend à ce qu'on lui fasse rapport de façon officielle et officieuse.

Le cardinal surveille les activités de la CNF grâce à une combinaison de postes administratifs et de réseaux clientélistes, une caractéristique clé de la formation de l'État durant cette période. Les six représentants qui proposent la création de la compagnie, à la demande du cardinal, entretiennent des liens étroits avec lui. Outre Richelieu lui-même, les associés les plus éminents sont ses clients, dont Isaac et Claude de Razilly, Philippe de

76. BAC, MC, v. L, étude LI, $\mathrm{n}^{\circ}$ 86, “Convention entre le duc de Ventadour et Jean de Lauson pour le paiement de la charge du vice-roi du Canada», 29 juin 1627; BAC, Fonds du Ministère des affaires étrangères, Memoire et documents, France, v. 785, f. 178, Lauson à Richelieu, 30 juin 1627.

77. Un arrêt royal concernant la liquidation des dettes de la CNF comprend une somme due à la Compagnie de Montmorency pour la perte de privilèges de commerce. Il note que Richelieu a renoncé à son droit à la part du vice-roi dans la plus vieille compagnie, un droit qu'il avait acheté de Ventadour. Voir BAC, Série E, Conseil d'Etat du Roi, v. 181c, fo. 144-152, 24 juillet 1643; L. Campeau, Les Finances publiques..., op. cit., 41; L.-A. Boiteux, Richelieu, grand maitre..., op. cit., 148, 278.

78. "Articles et conventions de société et compagnie du 7 mai 1627 ", dans Édits, ordonnances royaux, article 26 .

79. G. Carpin, Le Réseau du Canada..., op. cit., 88. 
Longvilliers, sieur de Poincy, Sébastien Cramoisy, Gabriel Lattaignant et Louis Houel; d'autres associés sont des dirigeants clés dans l'administration navale et financière, qui appuient le projet par conviction ou par suite de pressions ${ }^{80}$. Certains clients du cardinal agissent comme informateurs lors de leurs voyages à travers la France; d'autres reçoivent des commissions pour mener certaines affaires au nom de Richelieu. Plusieurs ont des liens avec plus d'une compagnie établie sous le grand maître ${ }^{81}$. Ce dernier point évoque la possibilité que ces hommes voient un avantage à participer à plusieurs des projets de leur protecteur: cela multiplie les occasions de recevoir des honneurs et des faveurs, tout en offrant la possibilité de compenser les entreprises infructueuses avec les profits des autres. Pour le cardinal, avoir des protégés dans ces compagnies lui permet de surveiller de près divers projets dans sa qualité officielle.

L'extension en Nouvelle-France de l'autorité maritime sous la Couronne a pour effet de remplacer une autorité quasi féodale par une autre. Comme Montmorency qui établit une nouvelle compagnie en devenant vice-roi, ainsi Richelieu remplace cette même compagnie par la sienne propre. Si la création de la CNF est le résultat de plusieurs facteurs, l'un d'entre eux se démarque comme facteur crucial: le désir d'exercer un contrôle sur les membres de la compagnie. La mobilisation de sa propre clientèle a pour corollaire le blocage de celle de ses rivaux. Ayant des liens étroits avec l'amirauté et Montmorency, le chef de l'ancienne compagnie, de Caen, entretient des liens avec l'institution et il est en mesure de remettre en question les projets maritimes du cardinal ${ }^{82}$. Le coût de la

80. Isaac de Razilly est le premier capitaine de la marine du Ponant, chef d'escadre et conseiller de Richelieu sur les affaires maritimes; Claude de Razilly est capitaine et plus tard vice-amiral de l'amirauté française; Philippe de Longvillliers, sieur de Poincy, un chevalier de Malte, deviendra gouverneur de Saint-Christophe aux Indes occidentales; Sébastien Cramoisy est un imprimeur éminent, libraire et éditeur des Relations des Jésuites; Gabriel Lattaignant est un ancien maire de Calais; et Louis Houel est conseiller du roi et contrôleur général des salines à Brouage. Parmi les agents royaux dans la CNF, on retrouve le surintendant des finances, le marquis d'Effiat, et l'intendant de la marine, Martin de Mauvoy, l'un des clients du cardinal. Pour la liste complète des membres, voir l'annexe «A» dans $\mathrm{M}$. Trudel, HNF..., op. cit., 3,1:415-425.

81. G. Carpin, Le Réseau du Canada..., op. cit., 63-64, 73, 98; P. Castagnos, Richelieu face à la mer..., op. cit., 129-141.

82. De Caen a au moins un autre allié bien placé dans l'amirauté de France, le sieur de Saint Martin, lieutenant-général de l'amirauté à Paris, qui se porte garant pour lui dans le cadre d'une entente signée en 1626 et portant sur la Compagnie de Montmorency. Voir BAC, MC, v. CI, étude CV, n 267,31 mars 1626; BAC, Série E, Conseil d’Etat du Roi, v. 87a, f. 41, 4 avril 1626. Dans le cadre d'une longue affaire portant sur l'indemnisation due pour la révocation précoce du contrat de la Compagnie de Montmorency, la CNF accuse de Caen d'avoir obtenu des privilèges de commerce uniquement "par la faveur de quelques personnes qui avaient crédit près du seigneur de Montmorency ». BAC, Collection Margry, v. 9269, fo. 276, 16 août 1629. Sur le désir de Richelieu de contrôler la compagnie, voir G. Carpin, Le Réseau du Canada..., op. cit., 78 . 
révocation des privilèges de la compagnie de Montmorency est élevé : il impose un fardeau financier important à la CNF durant les premières années de la nouvelle compagnie ${ }^{83}$. Pour la Couronne et pour Richelieu personnellement, l'incorporation de la Nouvelle-France au domaine maritime du roi vaut bien le poids de l'indemnisation versée.

\section{CONCLUSION}

L'histoire transatlantique de la mise en place de la CNF démontre que la souveraineté outre-mer et le pouvoir dans la métropole sont les deux faces d'une même pièce. Du point de vue du roi comme de celui de l'amiral, puis du grand maître, la lutte pour le contrôle de la mer, au début du XVII ${ }^{e}$ siècle, ne se limite pas aux côtes de la France, elle s'étend au contraire jusqu'en Nouvelle-France. La toute nouvelle colonie, plus imaginée que réelle, est intéressante pour le contrôle qu'elle offre sur les routes commerciales maritimes et sur les déplacements des sujets français. Ainsi, c'est Montmorency qui, pour la première fois, incorpore la Nouvelle-France à une institution métropolitaine. Des postes d'amirauté comme celui d'intendant sont créés en Nouvelle-France et deviennent un élément durable de l'administration coloniale. L'achat d'une vice-royauté fait partie de l'effort global de Montmorency pour étendre l'autorité de l'amirauté - et, par extension, de la Couronne - sur tous les ports de France. Richelieu, par la suite, travaille selon la même logique dans l'application de la grande maîtrise. Contrairement aux arguments souvent invoqués, les colonies n'étaient pas des lieux à part, mais des éléments intégrants d'un programme maritime qui prenait comme modèle les compagnies néerlandaises et anglaises dans le but de revitaliser le commerce et la navigation. Le contrôle royal des affaires maritimes s'accroît sous l'administration de l'un et de l'autre, alors que ces hommes renforcent leur autorité personnelle. Chacun cherche à écarter les concurrents, à remplacer ceux-ci par des clients fidèles ou des parents et à profiter des avantages privés qu'on peut amasser au service du roi. Les activités outre-mer figurent dans les calculs des destins dynastiques et politiques de la même façon que celles de la métropole. Une approche transatlantique repositionne l'histoire des origines de la CNF dans le contexte des rivalités personnelles et institu-

83. Le recours en dédommagement déposé par Guillaume de Caen et ses associés se déroule de 1627 à 1634. Le montant finalement accordé est de 79900 livres, dont seulement 19501 auront été versées en 1642. Voir BnF, dépt. de ms., fonds français, v. 16738, f. 44-67, 27 août 1634; BAC, MC, v. I, étude XVI, n 72, 23 février 1636; BAC, C11A, corresp. gén., f. 195-209v, «Etat général des debtes passives de la Compagnie générale de la Nouvelle-France», 1641-1642. 
tionnelles pour le contrôle du commerce maritime et de la navigation en France. Cette perspective souligne non seulement la relation intime entre la formation de l'État et la création d'un empire, mais également l'imprévu et la contestation associés à ces processus au début du XVII ${ }^{\mathrm{e}}$ siècle.

Traduction: Hélène Paré et Steven Watt 